\title{
Sind Gymnasiastinnen und Gymnasiasten mit mehr Grit erfolgreicher?
}

\section{Untersuchung der Zusammenhänge zwischen Grit, selbstreguliertem Lernen und Lernerfolg}

\author{
Yves Karlen $(\mathbb{D} \cdot$ Katharina Maag Merki · Carmen Hirt • Francesca Suter
}

Online publiziert: 11 . Oktober 2018

(C) Der/die Autor(en) 2018

Zusammenfassung Grit umfasst die Fähigkeit, längerfristige Ziele trotz Herausforderungen mit Beharrlichkeit und beständigem Interesse zu verfolgen. Dieser Fähigkeit wird das Potenzial zugesprochen, interindividuelle Leistungsunterschiede zu erklären. In dieser Studie haben wir den Zusammenhang zwischen Grit, dem selbstreguliertem Lernen (SRL) und der Leistung im Kontext des Verfassens einer längerfristigen und anspruchsvollen schriftlichen Arbeit, der Maturarbeit, untersucht. Hierfür wurden 1215 Schülerinnen und Schüler der gymnasialen Oberstufen (11./12. Klasse) während des einjährigen Erstellungsprozesseses der Maturaarbeit mehrmals befragt. Die multivariaten Analysen zeigen auf, dass Beharrlichkeit metamotivationales Wissen, motivationale Regulationsstrategien und kognitive Regulationskompetenzen vorhersagt. Beständiges Interesse korreliert positiv mit den kognitiven Regulationskompetenzen, geht jedoch mit einer geringen selbstberichteten Nutzungsangabe von motivationalen Regulationsstrategien einher. Von den beiden Grit Dimensionen sagte lediglich Beharrlichkeit die Maturaarbeitsnote ohne und mit Kontrolle der SRL-Kompetenzen voraus. Die Ergebnisse dieser Studie verdeutlichen, dass für die Erreichung von anspruchsvollen Zielen neben kognitiven Kompetenzen

\footnotetext{
Y. Karlen $(\bowtie) \cdot$ C. Hirt

Institut Sekundarstufe I \& II, Pädagogische Hochschule FHNW, Bahnhofstrasse 6, 5210 Windisch, Schweiz

E-Mail: yves.karlen@fhnw.ch

C. Hirt

E-Mail: carmen.hirt@fhnw.ch

K. Maag Merki · F. Suter

Institut für Erziehungswissenschaft, Universität Zürich, Freiestrasse 36, 8032 Zürich, Schweiz

K. Maag Merki

E-Mail: kmaag@ife.uzh.ch

F. Suter

E-Mail: francesca.suter@ife.uzh.ch
} 
auch die Fähigkeit, diese Kompetenzen über die Zeit hinweg beharrlich einzusetzen, unterstützend ist. Zudem weisen die Ergebnisse darauf hin, dass sich die beiden Grit Dimensionen in ihrer Vorhersagkraft voneinander unterscheiden.

Schlüsselwörter Grit · selbstreguliertes Lernen · Motivation · metakognitives Wissen · Lernstrategien

\title{
Are grittier college students more successful?
}

Investigating grit and it's relation to self-regulated learning and academic achievement

\begin{abstract}
Grit entails working strenuously and staying highly interested even in the face of setbacks in order to reach long-term goals. Grit has been discussed as potential predictor and determinant of academic performance. The present study investigated the relationship between grit, self-regulated learning (SRL), and achievement in the context of writing a larger and complex scientific paper, the "Maturaarbeit". We surveyed several times 1215 upper secondary school students (grad 11/12; ISCED level 3) during the one-year period of writing the Maturaarbeit. Structural equation modeling revealed perseverance of effort as a consistent predictor for all indicators of SRL including metamotivational knowledge, motivational regulation strategies, and cognitive regulation competencies. Consistency of interest positively correlated with cognitive regulation competencies, however, showed a negative correlation on the self-reported use of motivational regulation strategies. Further, we found significant indirect effects of grit on students ' achievement above SRL competencies. However, only perseverance of effort predicted achievement before, and after accounting for SRL. The results of this study indicated that, in addition to cognitive skills and the engagement in learning activities, the ability to use these skills persistently over time is supporting the achievement of challenging goals. Further, the result indicated that perseverance of effort and consistency of interest showed different incremental validity.
\end{abstract}

Keywords Grit · Self-regulated learning · Motivation · Metacognitive knowledge · Learning strategies

\section{Einleitung}

Das Erstellen einer umfangreichen schriftlichen Arbeit erfordert eine Vielzahl an Kompetenzen. Neben fachspezifischen kognitiven Fähigkeiten tragen insbesondere Anstrengungsbereitschaft und Persistenz sowie Kompetenzen zum selbstregulierten Lernen (SRL) bedeutsam zum Lernerfolg bei (Credé und Phillips 2011; Graham und Harris 2000; Karlen 2017). Die Fähigkeit, längerfristige Ziele mit Interesse und Beharrlichkeit zu verfolgen und sich dabei von Herausforderungen und Rückschlägen nicht entmutigen zu lassen, wird unter dem Begriff Grit subsummiert (Duckworth et al. 2007). Grit wird das Potenzial zugeschrieben, Performanz- und Leistungsunterschiede zwischen Individuen mit vergleichbaren kognitiven Fähigkeiten zu erklären 
(Duckworth und Quinn 2009). Obwohl in verschiedenen Studien Zusammenhänge zwischen Grit und Leistung aufgezeigt wurden, erweisen sich diese zugleich als inkonsistent (Bowman et al. 2015; Dumfart und Neubauer 2016; Steinmayr et al. 2018). Die Beziehung zwischen Grit und Leistung wird einerseits durch die Performanzsituation wie beispielsweise durch den Schwierigkeitsgrad und die Dauer der Aufgabe, und andererseits durch die individuellen SRL-Kompetenzen beeinflusst (Credé et al. 2017; Wolters und Hussain 2015). Die vorliegende Studie knüpft an diese Befunde und hat zum Ziel, die Effekte von Grit auf das SRL und die Leistung $\mathrm{zu}$ untersuchen. Im Fokus steht dabei die für den Abschluss des Gymnasiums relevante Maturaarbeit, eine anspruchsvolle und umfangreiche schriftliche Arbeit, die die Schülerinnen und Schüler am Ende des Gymnasiums im Verlaufe eines Jahres erstellen müssen.

\section{Theoretischer Hintergrund}

\subsection{Definition und Erfassung von Grit}

Grit beschreibt die Fähigkeit, das Interesse und das Engagement zur Erreichung von längerfristigen Zielen kontinuierlich und fokussiert aufrechtzuerhalten (Duckworth et al. 2007). Lernende mit mehr Grit halten in der Tendenz eher Verpflichtungen ein, übernehmen eher Verantwortung für das eigene Arbeitsverhalten und verfolgen Ziele persistent (Duckworth und Quinn 2009). Ferner umfasst Grit die Fähigkeit, Durchhaltevermögen und Ausdauer aufzubringen und auch bei Rückschlägen und Herausforderungen hart zu arbeiten. Entsprechend finden sich in der Literatur enge Zusammenhänge mit Konstrukten wie Gewissenhaftigkeit, Disziplin und Selbstkontrolle (u.a. Credé et al. 2017; Fleckenstein et al. 2014). Gemeinsam ist diesen, dass sie sich durch Attribute wie verantwortungsbewusst, zielstrebig, fleißig und selbstdiszipliniert beschreiben lassen. Im Vergleich zur Gewissenhaftigkeit und zur Selbstkontrolle betont Grit jedoch stärker die Aspekte Passion und Anstrengungsbereitschaft in Bezug auf das Erreichen von längerfristigen Zielen in herausfordernden Situationen, wodurch insbesondere auch das Streben nach Erfolg in Leistungssituationen über einen längeren Zeitraum in den Fokus rückt (Duckworth et al. 2007). Als solches besitzt Grit das Potenzial, Vorgehensweisen in unterschiedlichen Leistungskontexten zu beeinflussen (Duckworth und Quinn 2009).

Duckworth et al. (2007) haben Grit als zweidimensionales Konstrukt konzipiert, das aus den beiden Dimensionen Beharrlichkeit und beständiges Interesse besteht. Trotz der zweidimensionalen Struktur haben Duckworth und Kollegen eine Gesamtskala als integratives Maß zur Erfassung von Grit gebildet, die sich in ihren Studien als reliabel herausstellte und inkrementelle Vorhersagekraft in Bezug auf Bildungserfolg, Ausdauer und Engagement aufwies (siehe auch Duckworth und Quinn 2009). Während einige Forschergruppen Grit ebenfalls als Gesamtkonstrukt modellierten, weisen andere darauf hin, dass es sich bei Beharrlichkeit und beständigem Interesse zwar um korrelierte Grit Dimensionen handelt, diese jedoch über unterschiedliche Prädiktionskraft verfügen (Muenks et al. 2017; Steinmayr et al. 2018; Tyumeneva et al. 2017). Zum Zusammenhang zwischen den beiden Dimensionen finden sich 
zudem divergente Ergebnisse. Es werden sowohl eher starke Korrelationen (Arslan et al. 2013), als auch eher moderate und schwächere Zusammenhänge berichtet (Fleckenstein et al. 2014; Tyumeneva et al. 2017; Weisskirch 2016). In ihrer Metanalyse kommen Credé et al. (2017) zum Schluss, dass die beiden Grit Dimensionen im Durchschnitt eher stark miteinander korrelieren. Zugleich konnten sie aber aufzeigen, dass Beharrlichkeit über eine größere kriteriale Validität verfügt als beständiges Interesse, weshalb sie dafür plädieren, die beiden Dimensionen separat zu betrachten (siehe auch Steinmayr et al. 2018).

\subsection{Kompetenzen des selbstregulierten Lernens}

Das SRL stellt einen aktiven Prozess dar, in welchem Lernende verschiedene Selbstregulationsmaßnahmen kognitiver, metakognitiver, motivational-emotionaler oder verhaltensmässiger Art ergreifen, um Herausforderungen erfolgreich zu meistern und angestrebte Lernziele zu erreichen (Pintrich 2000). Hierbei überwachen, kontrollieren und regulieren Lernende ihre Kognitionen, ihre Motivation und ihr Verhalten in Abhängigkeit von diesen Zielen, den kontextuellen Rahmenbedingungen und der Aufgabenstellung. Entsprechend müssen Lernende über eine Vielfalt an SRLKompetenzen verfügen, um Aktivitäten, die der Initiierung und dem Aufrechterhalten des Lernens dienen, erfolgreich anzuwenden. Damit wird die Absicht verfolgt, das Lernverhalten vor, während und nach dem Lernprozess zu regulieren und die Informationsverarbeitung durch den Einsatz von kognitiven Lernstrategien zu steuern.

Kognitive Regulationskompetenzen umfassen den effektiven Einsatz von unterschiedlichen kognitiven Strategien, wobei sich diese wiederum auf das Transformieren, Elaborieren und Wiederholen von Informationen beziehen (Weinstein et al. 2011). Nota et al. (2004) fanden heraus, dass insbesondere kognitive Regulationskompetenzen einen signifikanten Prädiktor sowohl für die Abschlussnoten in unterschiedlichen Fächern der gymnasialen Oberstufe, als auch für die Leistung in verschiedenen Universitätskursen darstellen. Lernende, die Strategien mit einer hohen Qualität einsetzen und zudem auf ein tieferes Verständnis der Lerninhalte abzielen, sind erfolgreicher als Lernende, die eine eher oberflächliche Herangehensweise wählen und Defizite in der Nutzungsqualität aufweisen (Leopold und Leutner 2002).

Die Regulation des Lernens kann ein anstrengendes Unterfangen sein, bei welchem neben kognitiven Lernstrategien insbesondere motivationale Regulationskompetenzen und Ressourcen einen zentralen Stellenwert einnehmen (Wolters 2003). Lernende können auf unterschiedliche motivationale Regulationsstrategien (z.B. Steigerung der persönlichen Bedeutsamkeit, Selbstbelohnung) zurückgreifen, um ihre Motivation zu initiieren, aufrechtzuerhalten oder zu erhöhen (Schwinger et al. 2007). Der erfolgreiche Einsatz von motivationalen Regulationsstrategien beeinflusst in erste Linie die Anstrengungsbereitschaft, das Engagement und die Persistenz einer Person bei der Bearbeitung einer spezifischen Aufgabe. Aus diesem Grund werden hauptsächlich schwache bis moderate direkte oder lediglich indirekte Effekte der Nutzung von motivationalen Regulationsstrategien auf den Lernerfolg berichtet (Smit et al. 2017; Teng und Zhang 2018). Die Lernsituation und die damit verbundenen motivationalen Herausforderungen beeinflussen die Wahl und den Einsatz 
von motivationalen Regulationsstrategien (Engelschalk et al. 2015). Demnach ist anzunehmen, dass motivationale Regulationsstrategien eher bei anspruchsvollen und längerfristigen Aufgaben, deren erfolgreiche Bewältigung tatsächlich die Regulation der Motivation erfordert, einen Effekt auf die Leistung aufweisen.

Der Einsatz von Strategien setzt neben einem Mindestmaß an grundlegender Motivation auch Wissen über die anwendungsbezogenen Eigenschaften von Strategien voraus (Wolters 2003). Die effektive Nutzung von motivationalen Regulationsstrategien bedingt demnach spezifisches metakognitives Wissen über motivationale Aspekte des Lernens, d.h. metamotivationales Strategiewissen. Das metamotivationale Strategiewissen umfasst Wissen über die motivationalen Lernanforderungen und Eigenschaften von Aufgaben, die als solche mehr oder weniger motivierend sein können, Wissen über unterschiedliche Arten von motivationalen Regulationsstrategien sowie über anwendungsrelevante Informationen für deren zielgerichteten Einsatz (Paris et al. 1983). Es handelt sich folglich um bewusstseinsfähiges Wissen höherer Ordnung, das die Situations- und Aufgabenangemessenheit sowie die Nützlichkeit von verschiedenen motivationalen Regulationsstrategien enthält. Auf der Basis dieses konditionalen Wissens kann eine Person entscheiden, welche Strategie wann eingesetzt werden soll. Das metamotivationale Strategiewissen stellt somit eine wichtige Voraussetzung für die gezielte Auswahl von Strategien zur erfolgreichen Steuerung und Regulierung der eigenen Motivation dar (Karlen 2015; Wolters 2003). Obwohl verschiedene Studien die Bedeutsamkeit des metakognitiven Wissens für die Leistung in unterschiedlichen Domänen aufzeigen (Händel et al. 2013; Maag Merki et al. 2013; Neuenhaus et al. 2017), ist anzunehmen, dass das metamotivationale Strategiewissen vorwiegend eine Steigerung der Motivation zur Folge hat und somit eher einen indirekten als einen direkten Einfluss auf die Leistung nimmt (u. a. Lenzner und Dickhäuser 2011).

\subsection{Grit und selbstreguliertes Lernen}

Grit hat das Potenzial, individuelle Unterschiede beim SRL zu erklären, da Grit die Ausdauer und Leidenschaft eines Individuums umfasst, trotz Misserfolgen und Herausforderungen längerfristige Ziele zu verfolgen. Somit liegt diesem Konstrukt eine motivationale Antriebsfunktion und Überzeugung zu Grunde, die strategisches Verhalten begünstigen kann (Bowman et al. 2015; Duckworth et al. 2007; Hodge et al. 2017). Entsprechend fanden Duckworth et al. (2011) heraus, dass eine höhere Grit-Ausprägung mit einer aktiveren und strategischeren Vorgehensweise sowie einem höheren Engagement und folglich einer höheren Arbeitsqualität einhergeht. Forschungsbefunde zeigen zudem positive Zusammenhänge zwischen Grit und Metakognition auf. Grit konnte in verschiedenen Studien sowohl die Nutzung von metakognitiven Regulationsstrategien (Muenks et al. 2017; Wolters und Hussain 2015), als auch des metakognitiven Wissens vorhersagen (Arslan et al. 2013). Schmidt et al. (2017) zeigten bei einer Teilstudie mit Schülerinnen und Schülern der Sekundarstufe I auf, dass Lernende mit mehr Grit weniger prokrastinieren als Lernende mit einer geringeren Grit-Ausprägung. Dieser Zusammenhang zeigte sich ebenfalls bei Wolters und Hussain (2015), die Universitätsstudierende untersuchten und Grit nicht als Gesamtkonstrukt in ihr Modell integrierten, sondern die beiden 
Grit Dimensionen Beharrlichkeit und beständiges Interesse getrennt betrachteten. Sowohl Beharrlichkeit als auch beständiges Interesse korrelierten negativ mit der Prokrastination. Während Beharrlichkeit zudem sowohl positiv mit motivationalen, metakognitiven und kognitiven Strategien korrelierte, zeigte sich beim beständigen Interesse lediglich ein positiver Zusammenhang mit dem Zeit- und Lernumgebungsmanagement. Auch Bowman et al. (2015) fanden in ihrer Studie mit Universitätsstudierenden heraus, dass sich die beiden Grit Dimensionen in ihrer Vorhersagekraft hinsichtlich den SRL-Kompetenzen unterscheiden. Beharrlichkeit stellte sich als bedeutsamer Prädiktor für Persistenz, Studierfähigkeit, Studienzufriedenheit und Zugehörigkeitsgefühl heraus. Demgegenüber war beständiges Interesse negativ mit der Intention verbunden, einen Fachwechsel vorzunehmen. Über verschiedene Studien hinweg zeigten sich auch positive Zusammenhänge zwischen Grit (insbesondere Beharrlichkeit) und motivationalen Konstrukten wie Selbstwirksamkeit, Anstrengungsbereitschaft, Werthaltungen und Erfolgserwartung (Fleckenstein et al. 2014; Hodge et al. 2017; Muenks et al. 2017; Wolters und Hussain 2015) oder auch generellen motivationalen Maßen wie ,schulische Motivation“ (Eskreis-Winkler et al. 2014). Als mögliche Erklärung für den Zusammenhang zwischen Grit und Motivation werden in der Literatur insbesondere die konzeptionelle Überlappung zwischen Grit und anderen Konstrukten wie Gewissenhaftigkeit, Engagement und Persistenz diskutiert, die teils eng mit motivationalen Variablen korrelieren (Credé et al. 2017; Fite et al. 2017; Steinmayr et al. 2018). Gesamthaft wird deutlich, dass Lernende mit mehr Grit im Vergleich zu Lernenden mit weniger Grit persistenter sind, vermehrt Strategien einsetzen, eine höhere Motivation aufweisen, über mehr metakognitives Wissen verfügen und insbesondere eher dazu bereit sind, Zeit und Engagement für schulisches Lernen aufzuwenden.

\subsection{Grit und Leistung}

Lernende mit mehr Grit sind eher in der Lage, ihre Fähigkeiten zu aktivieren, eine höhere Persistenz bei Herausforderungen zu zeigen, sich von Rückschlägen weniger entmutigen zu lassen und längerfristige Ziele konsequenter zu verfolgen (Duckworth et al. 2007). Dementsprechend finden sich in der Literatur positive Korrelationen zwischen Grit und verschiedenen Erfolgsmassen Verbleib im Bildungssystem, höheren Bildungsabschlüssen und einer geringeren Anzahl von Berufswechseln (Bowman et al. 2015; Duckworth und Quins 2009). Schmidt et al. (2017) fanden in ihrer Studie heraus, dass Grit als Gesamtkonstrukt ein signifikanter Prädiktor für die Schulnoten in Gymnasien ist. Analog zu West et al. (2016), die eine positive Zunahme der Punktzahlen in einem Leistungstest von der vierten in die achte Klasse für Lernende mit mehr Grit aufzeigen konnten, fanden auch Eskreis-Winkler et al. (2014), dass Lernende, welche über mehr Grit verfügen, die High-School erfolgreicher abschließen als Lernende mit weniger Grit. Den positiven Zusammenhang zwischen Grit und akademischer Leistung konnten auch Hodge et al. (2017) bei Universitätsstudierenden feststellen. Anhand multipler Regressionsanalysen zeigte sich, dass insbesondere Beharrlichkeit über inkrementelle Validität verfügte (siehe auch Bowman et al. 2015; Weisskirch 2016). In der Studie von Muenks et al. (2017) konnte ebenfalls lediglich die Beharrlichkeit die Schulnoten voraussagen, wohinge- 
gen das beständige Interesse nicht mit der Leistung korrelierte. Auch Rimfeld et al. (2016) fanden bei einem nationalen Schulleistungstest am Ende der obligatorischen Schulzeit bei Jugendlichen in Großbritannien lediglich Zusammenhänge zwischen Beharrlichkeit und Schulnoten.

Andere Studien beobachteten hingegen nur schwache oder keine Korrelationen zwischen Grit und Leistung, insbesondere nachdem verschiedene Kontrollvariablen in die Modelle eingefügt wurden. Nachdem Dumfart und Neubauer (2016) bei 500 Schülerinnen und Schülern der Sekundarstufe II Intelligenz und Gewissenhaftigkeit kontrollierten, verschwanden die Zusammenhänge zwischen Grit und den Leistungen in Sprache, Naturwissenschaften und dem Gesamtnotendurchschnitt über alle Fächer hinweg. Bei Bazelais et al. (2016) verschwand der Zusammenhang zwischen Grit und den Leistungen von Studierenden, nachdem sie die Vorleistungen kontrolliert hatten. Wolters und Hussain (2015) beobachteten zwar einen positiven Zusammenhang zwischen Beharrlichkeit und den Leistungserwartungen bei Universitätsstudierenden, dieser positive Zusammenhang verschwand jedoch, nachdem SRL-Kompetenzen als weitere Prädiktoren ins Regressionsmodell eingeführt wurden. Steinmayr et al. (2018) berichteten in ihrer Studie mit Gymnasiastinnen und Gymnasiasten über kleine Effekte von Grit (insbesondere Beharrlichkeit) auf die Leistung, nachdem sie Persönlichkeitsmerkmale, Motivation und Engagement kontrollierten. Die verschiedenen Ergebnisse machen deutlich, dass SRL-Kompetenzen und motivationale Variablen den Zusammenhang zwischen Grit und Leistung mediieren können.

Obwohl inkonsistente Befunde zum Zusammenhang von Grit und Leistung vorhanden sind, kommen Credé et al. (2017) in ihrer Metaanalyse zum Schluss, dass Personen mit mehr Grit in der Tendenz höhere Lernerfolge aufweisen als Personen mit weniger Grit. Vorwiegend Beharrlichkeit vermochte Varianzanteile der Leistung aufzuklären, während beständiges Interesse diesbezüglich eine untergeordnete Rolle einnahm. Gesamthaft ist die Erklärungskraft von Grit mit durchschnittlich knapp $4 \%$ Varianzaufklärung bescheiden. Mögliche Erklärungsansätze für die inkonsistenten Befunde könnten im Schwierigkeitsgrad und der Klarheit der Aufgabe zu finden sein: Bei klar definierten Aufgaben herrscht im Gegensatz zu diffusen Aufträgen Zielklarheit, wodurch längerfristige Ziele einfacher verfolgt werden können. Zudem wurden in bisherigen Untersuchungen im schulischen Kontext oftmals Schulnoten beigezogen. Diese schließen auch zu leichte oder kleinschrittige Aufgaben mit ein, die tendenziell wenig Grit erfordern. Deshalb ist anzunehmen, dass das Potenzial von Grit vorwiegend bei herausfordernden und längerfristigen Tätigkeiten zum Tragen kommt (Abuhassan und Bates 2015).

\section{Ziele der Studie}

Um das Gymnasium in der Schweiz erfolgreich abzuschließen, müssen Schülerinnen und Schüler gegen Ende ihrer gymnasialen Ausbildung eine wissenschaftspropädeutische Arbeit, die Maturaarbeit, verfassen. Die Maturaarbeit ist eine umfangreiche, schriftliche Arbeit, die innerhalb eines vorgegebenen Zeitraums, in der Regel innerhalb eines Jahres, vorwiegend in der außerschulischen Zeit eigenständig verfasst 
wird. Sie ist ein erforderlicher Bestandteil, um die Maturität (ähnlich dem Abitur) zu erlangen und damit Zugang zur universitären Bildung zu erhalten. In dieser Studie soll der Zusammenhang zwischen Grit und der Leistung bei der Maturaarbeit unter Berücksichtigung von SRL-Kompetenzen als mediierende Faktoren analysiert werden. Drei Fragestellungen stehen im Zentrum dieser Untersuchung:

1. Inwiefern bestehen Zusammenhänge zwischen Grit und verschiedenen SRL-Kompetenzen im Kontext einer umfangreichen und komplexen schriftlichen Arbeit? Analog den bisherigen Befunden gehen wir davon aus, dass Beharrlichkeit und beständiges Interesse die kognitive Regulationskompetenzen, die motivationale Regulationsstrategien und das metamotivationale Wissen signifikant vorhersagen (H1).

2. Inwiefern verfügt Grit über Prädikationskraft zur Vorhersage der Maturaarbeitsnote? Obwohl die Beziehung zwischen Grit und Leistung nur bedingt konsistent ist, nehmen wir an, dass Grit im Kontext einer umfangreichen und anspruchsvollen Aufgabe ein bedeutsamer Prädiktor darstellt. Im Spezifischen ist zu erwarten, dass Beharrlichkeit eine signifikante Vorhersagekraft besitzt, wohingegen dem beständigen Interesse eine untergeordnete Rolle zugeschrieben wird (H2).

3. Inwiefern mediieren SRL-Kompetenzen die Beziehung zwischen Grit und Leistung bei einer anspruchsvollen und umfangreichen Aufgabe? Basierend auf vorhergehenden Studien, die aufzeigten, dass verschiedene kognitive Fähigkeiten den Effekt von Grit auf die Leistung mediieren, gehen wir davon, dass sich indirekte Effekte von Grit über die SRL-Kompetenzen auf die Leistung zeigen (H3).

\section{Methode}

\subsection{Erhebungsdesign und Stichprobe}

Die dieser Studie zugrundeliegende Datenbasis stammt aus einer längsschnittlichen Erhebung, im Verlauf derer Schülerinnen und Schüler innerhalb eines Jahres zu insgesamt fünf Messzeitpunkten zum Erstellungsprozess ihrer Maturaarbeit befragt wurden ( $\mathrm{t} 1$ vor dem Start der Maturaarbeit, t2 nach Abgabe des Konzeptes, t3 während des Erstellungsprozesses, $\mathrm{t} 4$ nach Abgabe der Maturaarbeit, t5 nach der Präsentation der Maturaarbeit). Die Schülerinnen und Schüler der gymnasialen Oberstufe (11./12. Schuljahr) stammen aus zwölf verschiedenen städtischen und ländlichen sowie kleineren und größeren Gymnasien der Deutschschweiz. Die Beteiligung der Gymnasien an der Längsschnittstudie war freiwillig. Die Online-Befragungen waren jedoch für alle Schülerinnen und Schüler, die kurz vor Beginn ihrer Maturaarbeit standen, verpflichtend.

Die vorliegenden Analysen basieren auf der Gesamtstichprobe von $N=1215$ Schülerinnen und Schüler (56,9\% weiblich). Die Schülerinnen und Schüler waren zum ersten Messzeitpunkt im Mittel $\mathrm{M}=17,5$ Jahre $(\mathrm{SD}=0,80)$ alt. Zum vierten Messzeitpunkt waren die Schülerinnen und Schüler ein Jahr älter $(M=18,5$; $\mathrm{SD}=0,82) .83,4 \%$ der Schülerinnen und Schüler gaben Deutsch/Schweizerdeutsch 
als Muttersprache an. Die Stichprobe ist für die Schulstufe in Bezug auf die Geschlechterverteilung und die Herkunft repräsentativ.

\subsection{Instrumente}

Die Daten für die vorliegende Studie wurden mittels standardisierten Online-Befragungen erfasst. Zur Erfassung von Grit (t1), kognitiven Regulationskompetenzen (t3) und motivationalen Regulationsstrategien (retrospektiv, t4) wurde ein standardisierter Fragebogen eingesetzt. Demgegenüber erfolgte die Erfassung des metamotivationalen Strategiewissens (t3) anhand eines Wissenstests.

Grit wurde mit einer validierten deutschen Übersetzung der englischsprachigen Grit Skala von Duckworth und Quinn (2009) erfasst (Schmidt et al. 2017), die je vier Items zu den beiden Dimensionen Beharrlichkeit (z. B. „Alles was ich beginne, bringe ich auch zu Ende.“) und beständigem Interesse (z. B. „Ich setze mir oft ein Ziel, entscheide mich dann aber später doch, ein anderes Ziel zu verfolgen.“, recodiert) beinhaltet. Eine konfirmatorische Faktorenanalyse (KFA) mit Mplus (Version 8.1; Muthén \& Muthén, 1998-2017) wies zuerst unzufriedene Fit-Werte für ein Modell mit zwei korrelierenden Faktoren erster Ordnung auf. Zudem wies das Item „Von Rückschlägen lasse ich mich nicht entmutigen“ schwache Ladungen auf den latenten Faktor Beharrlichkeit auf (dieses Problem zeigte sich auch bei Bowman et al. 2015; Muenks et al. 2017). Da das Überwinden von Rückschlägen ein wichtiger Bestandteil des Beharrlichkeitskonstrukts darstellt, wurde das Item jedoch nicht ausgeschlossen. Analog zu Schmidt et al. (2017) wurden beim Konstrukt Beharrlichkeit Korrelationen der Residuen zugelassen. Zusätzlich wurden, basierend auf den Modifikationshinweisen in Mplus, die Korrelationen der Residuen zweier weiterer Items beim Konstrukt Beharrlichkeit und zweier Items beim beständigen Interesse erlaubt. Aufgrund der Ähnlichkeiten der jeweiligen Items, machten diese Änderungen inhaltlich Sinn. Das entsprechend modifizierte zweidimensionale Modell mit zwei korrelierten latenten Faktoren wies nun gute FitWerte auf: $\chi^{2}(16)=31.869$, RMSEA =0,028 (90 CI [0,013, 0,043]), CFI=0,993, $\mathrm{SRMR}=0,021$. Kontrastierend wies das eindimensionale Modell unzufriedenstellende Fit-Werte auf: $\chi^{2}(17)=224.994$, RMSEA =0,100 (90 CI $[0,089,0,112]$ ), $\mathrm{CFI}=0,911, \mathrm{SRMR}=0,069$. Aufgrund dieser Ergebnisse werden die Berechnungen mit dem zweidimensionalen Modell, welches zwei korrelierte latente Faktoren beinhaltet, fortgesetzt. Deskriptive Kennwerte der beiden Skalen sind der Tab. 1 zu entnehmen.

Kognitive Regulationskompetenzen. In Anlehnung an (Karlen, Suter und Compagnoni in Vorb.) wurde eine Skala zur Erfassung der selbsteingeschätzten Kompetenzüberzeugung, kognitive Strategien mit einer hohen Qualität anwenden zu können, eingesetzt, welche aus vier Items (z. B. ,Ich kann umfangreiche Themengebiete gut auf das Wesentliche reduzieren.") besteht. Einleitend wurden die Schülerinnen und Schüler gebeten, bei der Beantwortung der Items an die Maturaarbeit zu denken: „Wenn Sie an Ihrer Maturaarbeit denken: Inwiefern treffen die folgenden Aussagen auf Sie zu?“. Die Skala umfasst die Kompetenz, erfolgreiche Beziehungen und Verknüpfungen zwischen unterschiedlichen Inhalten herzustellen und diese logisch aufeinander zu beziehen, Themengebiete gut auf das Wesentliche zu reduzieren so- 
Tab. 1 Deskriptive Kennwerte der erfassten Konstrukte und deren Interkorrelationen

\begin{tabular}{lllllll}
\hline Konstrukte & 1 & 2 & 3 & 4 & 5 & 6 \\
\hline 1. Beharrlichkeit & - & $0,31^{* * *}$ & $0,20 * * *$ & $0,31 * * *$ & $0,15^{* * *}$ & $0,20^{* * *}$ \\
2. Beständiges Interesse & & - & $0,18^{* * *}$ & 0,04 & $0,07 *$ & $0,09 *$ \\
3. Kognitive Regulations- & & & - & $0,16^{* * *}$ & $0,16^{* * *}$ & $0,14^{* * *}$ \\
kompetenzen & & & & & \\
4. Motivationale Regulations- & & & & & $0,25 * * *$ & $0,11^{* * *}$ \\
strategien & & & & & \\
5. Metamotivationales Wissen & & & & & & 0,02 \\
6. Maturaarbeitsnote & & & & & & - \\
Mittelwert & 3,99 & 3,63 & 4,22 & 3,82 & 0,65 & 5,18 \\
Standardabweichung & 0,87 & 0,80 & 0,76 & 0,81 & 0,20 & 0,60 \\
Reliabilität & 0,72 & 0,71 & 0,83 & 0,64 & 0,89 & - \\
Beobachteter Range & $1-6$ & $1-6$ & $1-6$ & $1-6$ & $0-1$ & $2-6$ \\
Möglicher Range & $1-6$ & $1-6$ & $1-6$ & $1-6$ & $0-1$ & $1-6$ \\
\hline
\end{tabular}

$N=727-1101$

$* * * p<0,001, * * p<0,01, * p<0,05$

wie neue Informationen erfolgreich mit dem Vorwissen zu verknüpfen. Die Skala wies eine gute Reliabilität auf (siehe Tab. 1).

Motivationale Regulationsstrategien ${ }^{1}$ wurden mit vier Subskalen aus dem Messinstrument zur Erfassung der selbstberichteten Motivationsregulation von Schwinger et al. (2007) erhoben. Einleitend wurden die Schülerinnen und Schüler gebeten, sich auf die Maturaarbeit zu konzentrieren: „Der letzte Monat vor der Abgabe der Maturaarbeit kann manchmal sehr anstrengend sein. Deshalb setzen Schülerinnen und Schüler verschiedene Strategien ein, um die Arbeit fertigzustellen. Inwiefern treffen die folgenden Aussagen auf Sie zu?““. Erfasst wurden die Steigerung der persönlichen Bedeutsamkeit (3 Items, z. B. „Ich bemühte mich, das zu Lernende mit meinen eigenen Erfahrungen in Beziehung zu setzen.“), die Selbstbelohnung (4 Items, z. B. „Ich stellte mir selber irgendeine Belohnung in Aussicht, wenn ich die Arbeit erledigt habe."), die Umweltkontrolle (3 Items, z. B. „Ich sorgte dafür, dass so wenige Ablenkungen wie möglich auftreten.") und die Subskala Teilziele setzen (3 Items, z. B. „Ich ging die Aufgaben Schritt für Schritt an, um das Gefühl zu bekommen, gut voranzukommen."). Anhand der vier Subskalen wurde ein übergeordneter motivationaler Regulationsindex gebildet. Eine KFA in Mplus zeigte umgehend gute Fit-Werte für das eindimensionale Modell mit einem latenten motivationalen Regulationsindex: $\chi^{2}(1)=1,055$, RMSEA $=0,007$ [90 CI: 0,000, 0,082], CFI = 1,000, $\mathrm{SRMR}=0,006$. auf, so dass dieser Index folgend zur Anwendung kommt.

\footnotetext{
1 Um die empirische Distinktheit zwischen Grit und den motivationalen Regulationsstrategien zu belegen, wurden eine KFA gerechnet. Wie erwartet, zeigte ein unidimensionales Modell schlechte Fit-Werte auf: $\chi^{2}(50)=683,446, \mathrm{CFI}=0,793$, RMSEA =0,102 (90 CI [0,095, 0,109]), SRMR=0,087. Anhand des Modellvergleichs erwies sich das alternative Modell mit separaten aber korrelierten Konstrukten als geeigneter: $\chi^{2}(47)=105,060, \mathrm{CFI}=0,981, \mathrm{RMSEA}=0,032(90 \mathrm{CI}[0,024,0,040]), \mathrm{SRMR}=0,029$.
} 


\begin{tabular}{|c|c|c|c|c|c|c|}
\hline \multirow{2}{*}{$\begin{array}{l}\text { Stellen Sie sich vor, die Person hat momentan Mühe, mit der } \\
\text { Arbeit an der Maturaarbeit zu beginnen. Für wie nützlich } \\
\text { schätzen Sie die unten stehenden Vorgehensweisen ein, um } \\
\text { sich mit der Maturarbeit zu beschäftigen? }\end{array}$} & \multicolumn{6}{|c|}{$\begin{array}{l}1=\text { überhaupt nicht nützlich } \\
6=\text { sehr nützlich }\end{array}$} \\
\hline & 1 & 2 & 3 & 4 & 5 & 6 \\
\hline $\begin{array}{l}\text { a) Sie macht sich bewusst, wie wichtig es für sie ist, eine gute } \\
\text { Note zu bekommen. }\end{array}$ & & & & & & \\
\hline $\begin{array}{l}\text { b) Sie versucht, den Lernstoff in Bezug zu ihrem Alltag zu } \\
\text { setzen. }\end{array}$ & & & & & & \\
\hline $\begin{array}{l}\text { c) Sie nimmt sich vor, heute möglichst viel an der } \\
\text { Maturaarbeit zu machen. }\end{array}$ & & & & & & \\
\hline $\begin{array}{l}\text { d) Sie überlegt sich einen Weg, um das Arbeiten } \\
\text { unterhaltsamer zu machen. }\end{array}$ & & & & & & \\
\hline $\begin{array}{l}\text { e) Sie wartet, bis genügend Zeitdruck vorhanden ist, da sie } \\
\text { erst dann gut beginnen kann. }\end{array}$ & & & & & & \\
\hline $\begin{array}{l}\text { f) Sie sagt sich, dass sie nach der Arbeit etwas Schönes } \\
\text { machen wird, wenn sie jetzt damit beginnt. }\end{array}$ & & & & & & \\
\hline $\begin{array}{l}\text { g) Sie setzt sich für heute kein Lernziel, damit sie spontan } \\
\text { mehr oder weniger arbeiten kann. }\end{array}$ & & & & & & \\
\hline $\begin{array}{l}\text { h) Sie sagt sich, dass es nicht so schlimm ist, wenn sie heute } \\
\text { ein wenig in Verzug gerät. }\end{array}$ & & & & & & \\
\hline
\end{tabular}

Abb. 1 Beispielszenario aus dem metamotivationalen Strategiewissenstest

Metamotivationales Wissen (MeMoWi) wurde anhand eines neu entwickelten Wissenstests erfasst. Die Entwicklung des MeMoWi-Tests folgt in seiner Konstruktionsrationalen dem bereits bestehenden und vielfach validierten und erprobten szenariobasierten Verfahren zur Erfassung von domänen-spezifischem metakognitiven Strategiewissen (u. a. Händel et al. 2013; Maag Merki et al. 2013; Neuenhaus et al. 2017). Der MeMoWi besteht aus drei Szenarien, welche die drei Phasen des SRL (präaktionale, aktionale und postaktionale Phase) beim Erstellungsprozess der Maturaarbeit abbilden (siehe Einleitungstext der Abb. 1). Die befragten Schülerinnen und Schüler mussten bei jedem Szenario acht vorgeschlagene Strategien hinsichtlich ihrer Nützlichkeit zur Meisterung der szenariospezifischen Herausforderungen auf einer 6-stufigen Likertskala ( $1=$ überhaupt nicht nützlich bis $6=$ sehr nützlich) einschätzen (siehe Abb. 1). Die Auswertung des MeMoWi-Tests erfolgte analog anderer metakognitiver Wissenstests anhand von Paarvergleichen (z. B. Strategie X ist mehr oder weniger nützlich als Strategie Y). Dadurch wird das Wissen über die relative Nützlichkeit verschiedener Strategiealternativen bewertet. Zur Quantifizierung der inhaltlichen Validität dieser Paarvergleiche wurde im Vorfeld eine Expertenbefragung mit zehn Expertinnen und Experten aus dem universitären Umfeld durchgeführt, die über elaborierte Kenntnisse im Bereich Motivation verfügen. 
Bei der Expertenbefragung wurde die Übereinstimmung bezüglich der Nützlichkeit der dargebotenen Strategien und die Eindeutigkeit der daraus resultierenden Paarvergleiche untersucht. In die Auswertung gingen nur diejenigen Paarvergleiche ein, deren relative Nützlichkeit durch die Expertinnen und Experten zu mehr als $75 \%$ übereinstimmend beurteilt wurden. Um einen hohen Wissensscore zu erzielen, mussten die getesteten Schülerinnen und Schüler bei der Bearbeitung des MeMoWi im Sinne eines Qualitätsstandards zur selben Einschätzung gelangen wie die Expertinnen und Experten. Für jeden übereinstimmenden Paarvergleich erhielten die Schülerinnen und Schüler einen Punkt. Bei Nicht-Übereinstimmung mit dem Expertenrating wurden keine Punkte vergeben. Der Wissensscore wurde als Mittelwert über drei Szenarien und allen Paarvergleichen hinweg ermittelt. Der MeMoWiScore konnte einen Wert zwischen 0 (kaum metamotivationales Wissen resp. keine Übereinstimmung mit den Experten) und 1 (viel metamotivationales Wissen resp. hohe Übereinstimmung mit den Experten) annehmen. Der Wissenstest wies eine gute Reliabilität auf (siehe Tab. 1).

Leistungsmaß. Als Leistungsmaß für die Maturaarbeit wurde die offizielle Maturaarbeitsnote verwendet. Analog dem schweizerischen Bewertungssystem wies die Note eine mögliche Bandbreite von $1=\operatorname{sehr}$ schwach bis $6=$ ausgezeichnet auf.

\subsection{Statistische Analysen}

Die Daten wurden anhand deskriptiver Analysen sowie mittels Strukturgleichungsmodellen (SEM) in Mplus 8.1 (Muthén und Muthén 1998-2017) untersucht. Um die Effekte von Grit auf die SRL-Kompetenzen und die Leistung sowie mögliche indirekte Effekte zu überprüfen, wurde ein lineares SEM (Abb. 2) mit multiplen Mediationen modelliert und die indirekten Effekte nach der vorgeschlagenen bias-korrigierten Bootstrapping-Prozedur (10’000 Bootstrapping-Stichproben) nach MacKinnon et al. (2004) untersucht. Ist der Wert 0 beim $95 \%$ Konfidenzintervall nicht enthalten, gilt der indirekte Effekt als statistisch signifikant. Die Modellgüte wurde gemäss den Empfehlungen von Schermelleh-Engel et al. (2003) überprüft. Hierbei wurden verschiedene Kriterien zur Beurteilung der Güte von Strukturgleichungsmodellen berücksichtig. Ein sehr gutes Modell sollte $\chi^{2}$ Werte im Verhältnis zu den Freiheitsgraden von kleiner als drei, Comparative-Fit-Index (CFI) Werte grösser als 0,95, Root-Mean-Square-Error-of-Approximation (RMSEA) Werte kleiner als 0,05 und Standardized-Root-Mean-Square-Residual (SRMR) Werte kleiner als 0,06 aufweisen. Der Datensatz weist über alle Variablen hinweg durchschnittlich 4,3\% fehlende Werte (Range: 0,3-7,0\%) auf. Zur Einbeziehung aller fehlenden Werte wurde das Full-Information-Maximum-Likelihood-Schätzverfahren (FIML) verwendet. 


\section{Ergebnisse}

\subsection{Deskriptive Ergebnisse und Korrelationen}

Tab. 1 enthält die Mittelwerte, die Standardabweichungen und die Reliabilitätswerte aller Konstrukte sowie deren Interkorrelationen. Wie der Tab. 1 zu entnehmen ist, wurden alle Konstrukte reliabel erfasst. Die Korrelationskoeffizienten weisen auf schwache bis mittlere Zusammenhangseffekte zwischen den verschiedenen Konstrukten hin. Beharrlichkeit und beständiges Interesse sind mit $r=0,31$ moderat miteinander korreliert.

\subsection{Teilmodelle: Zusammenhänge zwischen Grit und Noten und Zusammenhänge zwischen SRL und Noten}

In einem ersten Schritt wurde die Vorhersagekraft von Grit auf die Leistung sowie die Vorhersagekraft der SRL-Kompetenzen auf die Leistung untersucht, um die separierten Effekte der Prädiktoren zu evaluieren. Dabei wurde zunächst ein Modell mit Beharrlichkeit und beständigem Interesse als Prädiktoren der Maturaarbeitsnote spezifiziert. Das entsprechende Modell wies mit $\chi^{2}(22)=62,931, \chi^{2} / \mathrm{df}=2,86$, $\mathrm{CFI}=0,983$, RMSEA $=0,039$ (90 CI $[0,028,0,050])$ und $\mathrm{SRMR}=0,029$ gute FitWerte auf. Wie angenommen, ist der Pfad von Beharrlichkeit $(\beta=0,22, p<0,001)$ auf die Leistung signifikant, wohingegen das beständige Interesse nicht zur Varianzaufklärung der Maturaarbeitsnote beitragen kann.

In einem zweiten Schritt wurde die Vorhersagekraft der SRL-Kompetenzen auf die Maturaarbeitsnote untersucht. Hierbei wurden Pfade vom metamotivationalen Wissen auf die motivationalen Regulationsstrategien und die kognitiven Regulationskompetenzen modelliert (beispielhaft in Abb. 2 zu sehen). Zudem wurden Pfade von den motivationalen Regulationsstrategien und den kognitiven Regulationskompetenzen auf die Maturaarbeitsnote spezifiziert. Das entsprechende Modell passte gut zu den Daten: $\chi^{2}(31)=60,037, \chi^{2} / \mathrm{df}=1,94, \mathrm{CFI}=0,982, \mathrm{RMSEA}=0,034$ (90 CI [0,021, $0,047]), \mathrm{SRMR}=0,043$. Es zeigten sich signifikante direkte Effekte des metamotivationalen Strategiewissens auf die motivationalen Regulationsstrategien $(\beta=0,31$, $p<0,001)$ und die kognitive Regulationskompetenzen $(\beta=0,19, p<0,001$.). Sowohl die motivationalen Regulationsstrategien $(\beta=0,12, p<0,01)$, als auch die kognitiven Regulationskompetenzen $(\beta=0,16, p<0,01)$ hatten einen direkten Effekt auf die Maturaarbeitsnote. Zudem zeigten sich signifikante indirekte Effekte des metamotivationalen Wissens über die motivationalen Regulationsstrategien und über die kognitiven Regulationskompetenzen auf die Maturaarbeitsnote.

\subsection{Gesamtmodell: Zusammenhänge zwischen Grit, SRL und Maturaarbeitsnote}

Das modellierte Gesamtmodell wies umgehend gute Modellfitwerte auf: $\chi 2(119)=$ 269,$195 ; \chi^{2} / \mathrm{df}=2,26, \mathrm{CFI}=0,966, \mathrm{RMSEA}=0,032$ (90 CI $\left.[0,027 ; 0,037]\right), \mathrm{SRMR}=$ 0,043. Abb. 2 enthält die standardisierten Koeffizienten der berechneten Pfade. Beharrlichkeit erwies sich als bedeutsamer Prädiktor für alle einbezogenen SRL-Kom- 


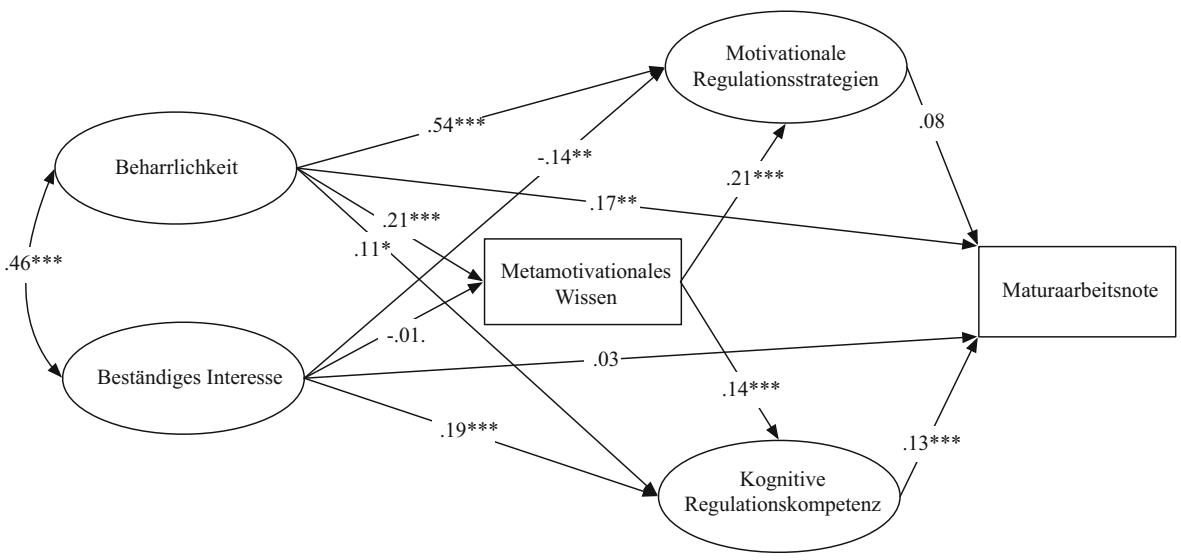

Abb. 2 Strukturgleichungsmodell zwischen Grit, selbstreguliertem Lernen und Maturaarbeitsnote. (Anmerkungen: $* * * p<0,001, * * p<0,01, * p<0,05)$

petenzen. Wie angenommen, weisen Schülerinnen und Schüler, die beharrlicher sind, ein höheres metamotivatonales Strategiewissen auf $(\beta=0,21, p<0,001)$, setzten mehr motivationale Regulationsstrategien ein $(\beta=0,54, p<0,001)$ und berichteten über höhere kognitive Regulationskompetenzen $(\beta=0,11, p<0,05)$ als Schülerinnen und Schüler mit einer geringeren Beharrlichkeitsausprägung. Während sich zwischen beständigem Interesse und motivationalen Regulationsstrategien ein negativer direkter Effekt $(\beta=-0,14, p<0,05)$ zeigte, das heißt, Schülerinnen und Schüler mit höherem beständigen Interesse weniger motivationale Regulationsstrategien einsetzten als Schülerinnen und Schüler mit geringerem beständigem Interesse, zeigte sich ein positiver Zusammenhang zu den kognitiven Regulationskompetenzen $(\beta=0,19, p<0,001)$. Zwischen beständigem Interesse und dem metamotivationalen Strategiewissen ergab sich kein signifikanter Zusammenhang. Wie angenommen und bereits im ersten Analyseschritt aufgezeigt, spielte das metamotivationale Wissen eine bedeutsame Rolle zur Vorhersage der selbstberichteten Strategienutzung. Sowohl der Pfad $(\beta=0,21, p<0,001)$ zu den motivationalen Regulationsstrategien, als auch der Pfad $(\beta=0,14, p<0,001)$ zu den kognitiven Regulationskompetenzen sind signifikant. Schülerinnen und Schüler mit höherem metamotivationalem Wissen berichteten folglich über höhere Kompetenzen im Bereich der motivationalen und kognitiven Strategienutzung. Des Weiteren zeigten sich Effekte auf die Maturaarbeitsnote. Schülerinnen und Schüler, die beharrlicher sind, erreichten bessere Noten $(\beta=0,17, p<0,01)$. Die Effektstärke von Beharrlichkeit auf die Noten verringert sich im Vergleich zu den Ergebnissen aus dem ersten Analyseschritt leicht. Zudem erzielten auch Schülerinnen und Schüler, die über höhere kognitive Regulationskompetenzen verfügten, bessere Maturaarbeitsnoten $(\beta=0,13, p<0,001)$. Der im ersten Analyseschritt dargelegte signifikante direkte Effekt der motivationalen Regulationsstrategien auf die Maturaarbeitsnote verliert hingegen seine Vorhersagekraft. Die im Modell eingefügten Variablen klärten 8,4\% Varianzanteile der Maturaarbeitsnote auf. 
Tab. 2 Schätzungen und Konfidenzintervalle der indirekten Effekte von Grit über die motivationalen Regulationsstrategien und über die kognitiven Regulationskompetenzen auf die Leistung

\begin{tabular}{llllll}
\hline Angenommene & \multicolumn{2}{l}{ Beobachtete Mediationseffekte } \\
& Estimate & SE & $95 \%$ CI & & Effektgrösse \\
& & & Tiefer & Höher & \\
\hline Beha $\rightarrow$ MR $\rightarrow$ MA & 0,035 & 0,030 & $-0,004$ & 0,073 & 0,042 \\
Beha $\rightarrow$ KR $\rightarrow$ MA & 0,012 & 0,012 & 0,001 & 0,025 & 0,015 \\
Best $\rightarrow$ MR $\rightarrow$ MA & $-0,012$ & 0,010 & $-0,026$ & 0,003 & $-0,011$ \\
Best $\rightarrow$ KR $\rightarrow$ MA & 0,027 & 0,013 & 0,007 & 0,047 & 0,025 \\
\hline
\end{tabular}

Signifikante indirekte (mediierte) Effekte sind kursiv.

Beha Beharrlichkeit, Best beständiges Interesse, $M R$ motivationale Regulationsstrategien, KR kognitive Regulationskompetenzen, $M A$ Maturaarbeitsnote

Tab. 3 Schätzungen und Konfidenzintervalle der indirekten Effekte von Grit über das metamotivationale Wissen, die motivationalen Regulationsstrategien und über die kognitiven Regulationskompetenzen auf die Leistung

\begin{tabular}{|c|c|c|c|c|c|}
\hline \multirow{3}{*}{$\begin{array}{l}\text { Angenommene } \\
\text { Mediationseffekte }\end{array}$} & \multicolumn{5}{|c|}{ Beobachtete Mediationseffekte } \\
\hline & \multirow[t]{2}{*}{ Estimate } & \multirow[t]{2}{*}{$\mathrm{SE}$} & \multicolumn{2}{|l|}{$95 \% \mathrm{CI}$} & \multirow[t]{2}{*}{ Effektgrösse } \\
\hline & & & Tiefer & Höher & \\
\hline $\mathrm{Beha} \rightarrow \mathrm{MW} \rightarrow \mathrm{MR} \rightarrow \mathrm{MA}$ & 0,003 & 0,002 & $-0,001$ & 0,006 & 0,003 \\
\hline $\mathrm{Beha} \rightarrow \mathrm{MW} \rightarrow \mathrm{KR} \rightarrow \mathrm{MA}$ & 0,003 & 0,002 & 0,001 & 0,006 & 0,004 \\
\hline Best $\rightarrow \mathrm{MW} \rightarrow \mathrm{MR} \rightarrow \mathrm{MA}$ & 0,000 & 0,001 & $-0,002$ & 0,001 & 0,000 \\
\hline Best $\rightarrow \mathrm{MW} \rightarrow \mathrm{KR} \rightarrow \mathrm{MA}$ & 0,000 & 0,001 & $-0,002$ & 0,002 & 0,000 \\
\hline
\end{tabular}

Signifikante indirekte (mediierte) Effekte sind kursiv markiert.

Beha Beharrlichkeit, Best beständiges Interesse, $M W$ metamotivationales Wissen, $M R$ motivationale Regulationsstrategien, $K R$ kognitive Regulationskompetenzen, $M A$ Maturaarbeitsnote

In den Tab. 2 und 3 sind die modellierten sowie empirisch bestimmten mediierten Effekte von Grit auf Leistung über die SRL-Kompetenzen aufgeführt. Wie der Tab. $2 \mathrm{zu}$ entnehmen ist, waren beharrlichere Schülerinnen und Schüler auch erfolgreicher bei der Maturaarbeit, weil sie über höhere kognitive Regulationskompetenzen verfügten. Dieser Effekt zeigte sich auch für das beständige Interesse. Schülerinnen und Schüler mit einem höheren beständigen Interesse verfügten über höhere kognitive Regulationskompetenzen und waren entsprechend erfolgreicher bei der Maturaarbeit. Wie in Tab. 3 aufgeführt, waren beharrlichere Schülerinnen und Schüler außerdem erfolgreicher bei der Maturaarbeit, weil sie neben höheren kognitiven Regulationskompetenzen auch über ein höheres metamotivationales Wissen verfügten.

\section{Diskussion}

Obwohl Grit das Potenzial zugeschrieben wird, sowohl interindividuelle Performanz- und Leistungsunterschiede als auch interindividuelle Differenzen beim SRL zu erklären (Duckworth et al. 2007), weisen verschiedene Studien und Metanalysen auf eine inkonsistente Befundlage hin (Credé et al. 2017). Es ist anzunehmen, dass 
die Aufgabenart hierbei eine wichtige Rolle spielt. Zu leichte und kleinschrittige Leistungsaufgaben erfordern weder Grit noch SRL-Kompetenzen, um erfolgreich gelöst zu werden. Zudem wurde in vergangenen Studien aufgezeigt, dass die Beziehung zwischen Grit und Leistung durch weitere Prädiktoren, wie zum Beispiel SRL-Kompetenzen, mediiert wird (Wolters und Hussain 2015). Bisherige Studien im schulischen Kontext erfassten oftmals die Schulnoten als Leistungsmaß, die auch Aufgaben einschließen, die kein Grit erfordern. Das Ziel dieser Studie war es deshalb, mögliche Zusammenhänge zwischen Grit, SRL-Kompetenzen und Leistung im Rahmen einer anspruchsvollen und umfangreichen schriftlichen Arbeit, der Maturaarbeit, zu untersuchen. Im Fokus standen drei Fragestellungen mit den folgenden Zielen: 1) Die Untersuchung des Zusammenhanges zwischen Grit und verschiedenen SRL-Kompetenzen im Kontext einer umfangreichen und komplexen schriftlichen Arbeit, 2) die Untersuchung der Prädiktionskraft von Grit zur Vorhersage der Maturaarbeitsnote sowie 3) die Untersuchung von Mediationseffekten von SRL-Kompetenzen auf die Beziehung zwischen Grit und Leistung.

\subsection{Grit als zweidimensionales Konstrukt}

Im Zentrum dieser Untersuchung stand die von Duckworth und Kollegen als Grit bezeichnete Fähigkeit eines Individuums, mit Beharrlichkeit und beständigem Interesse längerfristige Ziele zu verfolgen (Duckworth et al. 2007). Trotz der zweidimensionalen Struktur verwendeten Duckworth und Kollegen einen Gesamtscore, da sie einen übergeordneten Grit-Faktor bilden konnten (Duckworth et al. 2007; Duckworth und Quinn 2009). In den letzten Jahren wurde jedoch vermehrt darauf hingewiesen, dass die zweifaktorielle Struktur von Grit stärker zu berücksichtigen sei (u.a Fleckenstein et al. 2014). In Anlehnung an neuere Forschungsarbeiten (Credé et al. 2017; Steinmayr et al. 2018; Tyumeneva et al. 2017) konnte in der vorliegenden Studie die Zweidimensionalität des Konstruktes bestätigt werden. Beharrlichkeit und beständiges Interesse bilden zwei getrennte, aber korrelierte Faktoren. Aus diesem Grund wurde auf einen Gesamtscore verzichtet und Beharrlichkeit und beständiges Interesse als eigenständige Konstrukte in die Analysen eingefügt. Die Bedeutung dieses Vorgehens wird nachfolgend anhand der unterschiedlichen Prädiktionskräften von Beharrlichkeit und beständigem Interesse verdeutlicht.

\subsection{Zusammenhang zwischen Grit und selbstreguliertem Lernen}

Hinsichtlich der ersten Fragestellung zum Zusammenhang zwischen Grit und SRL konnten die Ergebnisse dieser Studie analog bisheriger Untersuchungsresultate (u. a. Muenks et al. 2017; Wolters und Hussain 2015) aufzeigen, dass Grit interindividuelle Unterschiede bei den SRL-Kompetenzen der Schülerinnen und Schüler zu erklären vermag. Schülerinnen und Schüler, die beharrlicher sind, berichteten über höhere kognitive Regulationskompetenzen, setzten mehr motivationale Regulationsstrategien ein und verfügten über ein höheres metamotivatonales Strategiewissen. Auch das beständige Interesse konnte zur Varianzaufklärung des SRL beitragen. Schülerinnen und Schüler mit einem höheren beständigen Interesse berichteten ebenfalls über höhere kognitive Regulationskompetenzen. Diese Befunde sind kongruent mit 
bereits beobachteten Zusammenhängen zwischen Grit und geringerer Prokrastination, höherer Selbstwirksamkeit, höherem wahrgenommenen Kompetenzerleben und vermehrter Strategienutzung (u.a. Schmidt et al. 2017; Weisskirch 2016; Wolters und Hussain 2015). Entgegen den Erwartungen zeigte sich jedoch ein negativer Zusammenhang zwischen dem beständigen Interesse und den motivationalen Regulationsstrategien. Schülerinnen und Schüler mit einem höheren beständigen Interesse berichteten über eine geringere Nutzung von motivationalen Regulationsstrategien. Dieses Ergebnis könnte sich dadurch erklären lassen, dass das beständige Interesse analog der Persönlichkeitseigenschaft Gewissenhaftigkeit positiv mit einer höheren akademischen Motivation zusammenhängt (De Feyter et al. 2012; Komarraju et al. 2009), wodurch der Bedarf, die eigene Motivation zu regulieren, um eine spezifische Tätigkeit auszuführen und aufrechtzuerhalten, entsprechend geringer ist und folglich zu einem geringeren motivationalen Regulationsstrategieeinsatz führt. Da mit einem beständigen Interesse ebenso eine höhere Motivation vorhanden ist, braucht es folglich keine regulierende Handlung, um die eigene Motivation absichtsvoll zu beeinflussen. Diese Annahme könnte auch den nicht vorhandenen Zusammenhang zwischen beständigem Interesse und dem metamotivationalen Wissen erklären. Da aufgrund einer höheren motivationalen Ausprägung weniger motivationale Regulationsstrategien eingesetzt werden, werden vermutlich weniger Erfahrungen gesammelt, um metamotivationales Wissen aufzubauen. Für den Erwerb und die Weiterentwicklung des metakognitiven Wissens sind jedoch unterschiedliche Nutzungserfahrung mit Strategien und die Reflexion des Strategieeinsatzes erforderlich (Artelt und Neuenhaus 2010). Zusammenfassend ist festzuhalten, dass Zusammenhänge zwischen Grit und SRL vorhanden sind. Wie vermutet (Hypothese 1) ist Grit ein bedeutsamer Prädiktor von SRL. Die beiden Grit Dimensionen unterscheiden sich jedoch in ihrer Vorhersagekraft voneinander. So hat sich vorwiegend Beharrlichkeit als inkrementell valide gegenüber SRL erwiesen. Dieses Resultat geht mit bisherigen Befunden einher, die ebenfalls die Unterschiede zwischen den beiden Grit Dimension bezüglich ihrer Vorhersagekraft des SRL hervorheben (u.a. Muenks et al. 2017; Wolters und Hussain 2015). Unsere Ergebnisse tragen dazu bei, interindividuelle Unterschiede beim SRL von Gymnasiastinnen und Gymnasiasten besser zu verstehen. Es könnte deshalb in Zukunft gewinnbringend sein, individuelle Dispositionen stärker in Modellen des SRL zu verankern.

\subsection{Prädiktionskraft von Grit zur Vorhersage der Maturaarbeitsnote}

Erwartungskonform mit der Hypothese 2 zum Zusammenhang von Grit und der Maturaarbeitsnote konnte in dieser Studie aufgezeigt werden, dass Grit Leistungsunterschiede zwischen Individuen über interindividuelle Differenzen bei den SRLKompetenzen zu erklären vermochte. Duckworth et al. (2007) gingen davon aus, dass beide Dimensionen maßgeblich zum Lernerfolg beitragen: Beständiges Interesse, weil es bedeutsam ist, längerfristige Ziele und Interessen persistent zu verfolgen und Beharrlichkeit, weil das Erlangen von Kompetenz ein großes Engagement erfordert und dabei auch mögliche Herausforderungen und Misserfolge überwunden werden müssen. Wie erwartet, deuten unsere Ergebnisse analog der Metaanalyse von Credé et al. (2017) darauf hin, dass sich die beiden Grit Dimensionen in ihrer Vor- 
hersagekraft zur Erklärung von Leistungsunterschieden voneinander unterscheiden. Während Beharrlichkeit einen signifikanten direkten Einfluss auf die Maturaarbeitsnote hatte, ließ sich ein direkter Effekt für das beständige Interesse nicht bestätigen (siehe auch Hodge et al. 2017; Steinmayr et al. 2018; Weisskirch 2016).

Neben direkten Effekten auf die Leistung werden Grit insbesondere indirekte Effekte auf die Leistung zugesprochen (für eine detaillierte Diskussion siehe Steinmayr et al. 2018). Wie entlang der Fragestellung 3 untersucht, zeigten sich indirekte Effekte von Grit über die SRL-Kompetenzen auf die Leistung (Hypothese 3). Beharrlichkeit führt zu erfolgreicheren Schülerinnen und Schüler, da diese Fähigkeit auch mit höheren SRL-Kompetenzen einhergeht. Beharrlichere Schülerinnen und Schüler verfügten sowohl über höheres metamotivationales Wissen, als auch über höhere kognitive Regulationskompetenzen, was sich positiv auf die Maturaarbeitsnote auswirkte. Schülerinnen und Schüler mit höherem beständigem Interesse berichteten über höhere kognitive Regulationskompetenzen, was sich wiederum positiv auf die Maturaarbeitsnote auswirkte. Die Bedeutung des beständigen Interesses könnte folglich darin liegen, dass vorwiegend die Erfolgserwartung oder der Wert einer Aufgabe erhöht wird. Durch eine auf diese Weise erhöhte Motivation wird zwar die Realisierung von Intentionen begünstigt, was sich direkt auf das Lernverhalten auswirken kann, aber eben lediglich einen indirekten Einfluss auf die Leistung ausübt (Wigfield und Eccles 2000; Wolters und Hussain 2015). Gesamthaft kann analog bisheriger Befunden in Studien, in denen das Leistungsmass eine anspruchsvollere Tätigkeit umfasst (Abuhassan und Bates 2015; Dumfart und Neubauer 2016), festgehalten werden, dass Grit für den Lernerfolg bedeutsam ist.

Die in dieser Studie berichteten Zusammenhänge zwischen Grit und Leistung sowie den SRL-Kompetenzen und Leistung bleiben auch bestehen, wenn in das in Abb. 2 spezifizierte Modell zusätzlich die Vornoten (Vorwissensmaß) der Schülerinnen und Schüler aus dem Fach/den Fächern, dem/den sie ihre Maturaarbeit thematisch zugeordnet haben, als Kontrollvariable eingefügt wird. Aufgrund der Vorhersagekraft der Vornote von $\beta=0,29(p<0,001)$ auf die Maturaarbeitsnote verringert sich die Effektstärke der Beziehung zwischen Beharrlichkeit und der Maturaarbeitsnote zwar leicht, bleibt aber signifikant positiv $(\beta=0,12, p<0,01)$. Es kann festgehalten werden, dass bei Schülerinnen und Schüler, die über vergleichbare leistungsbezogene Eingangsvoraussetzungen besitzen, diejenigen eine bessere Leistung bei der Maturaarbeit erzielten, die beharrlicher waren und über adaptivere SRLKompetenzen verfügten.

\subsection{Metamotivationales Wissen und Strategienutzung}

Nicht im Hauptfokus der vorliegenden Studie stehend, konnten die in anderen Untersuchungen berichteten Zusammenhänge zwischen dem metakognitiven Wissen und der Strategienutzung auf der gymnasialen Oberstufe bestätigt werden (u.a. Maag Merki et al. 2013). Der in dieser Studie eingesetzte metamotivationale Wissenstest ermöglichte es, domänenspezifisches Wissen über verschiedene Arten von motivationalen Regulationsstrategien und die Bedingungen ihres effektiven Einsatzes in spezifischen Lernsituationen im Rahmen des Verfassens einer Maturaarbeit reliabel und valide zu erfassen. Wie erwartet, konnte das metamotivationale Wissen 
interindividuelle Differenzen bei der selbstberichteten Nutzung von motivationalen Regulationsstrategien voraussagen. Analog zu den Befunden, die aufzeigen, dass metakognitives Wissen bis zu einem gewissen Grad generalisierbare und transferierbare Wissenselemente enthält (Neuenhaus et al. 2010), zeigte sich in unserer Studie zudem ein positiver Zusammenhang zwischen dem metamotivationalen Wissen und den kognitiven Regulationskompetenzen. Das metamotivationale Wissen stellt eine wichtige Bedingung für die Nutzung von Strategien dar (Karlen 2015). Da das metamotivationale Wissen in erster Linie zu einer erfolgreichen Regulation der Motivation beiträgt, konnten wir, wie vermutet, keinen direkten Effekt auf die Leistung nachweisen. Es zeigten sich jedoch signifikante indirekte Effekte des metamotivationalen Wissens über die Strategienutzung auf die Maturaarbeitsnote.

\subsection{Implikationen für die Praxis und weiterführende Forschungsfragen}

Die berichteten Zusammenhänge heben die Relevanz von Beharrlichkeit für den Erfolg beim Verfassen einer grösseren schriftlichen Arbeit hervor. Interventionsstudien konnten zeigen, dass sich Grit analog zu anderen Fähigkeiten und Eigenschaften verändern lässt (Eskreis-Winkler et al. 2018). Diesbezüglich nehmen die Lehrkräfte eine wichtige Vorbildfunktion ein, da sie als Rollenmodelle auf die Schülerinnen und Schüler positiv einwirken können (Duckworth et al. 2009). Lehrpersonen sollten den Schülerinnen und Schülern aufzeigen, wie sich die Fähigkeit entwickeln lässt, auch bei herausfordernden Aufgaben beharrlich zu bleiben und Wege zu suchen, wie sie Herausforderungen erfolgreich meistern können. Lehrpersonen könnten den Schülerinnen und Schüler beispielsweise zeigen, wie sie bei konkreten Aufgaben Teilziele setzen, welche die Realisierung der Aufgabe strukturieren und anhand derer die notwendigen Schritte zur längerfristigen Zielerreichung festzulegen sind. Dazu könnten längerfristige und komplexe Aufgaben mit klaren Zielen im Vorfeld der Maturaarbeit als wichtiges „Übungsfeld“ dienen. Schließlich weisen Interventionsstudien auch darauf hin, dass es hilfreich ist, wenn Schülerinnen und Schüler davon überzeugt sind, dass sich Fähigkeiten verändern lassen (Eskreis-Winkler et al. 2016). Entsprechende Überzeugungen könnten den Schülerinnen und Schülern helfen bei komplexen und längerfristigen Aufgaben auch bei Herausforderungen beharrlich zu bleiben.

\subsection{Limitationen und Ausblick}

Mit Blick auf die Limitationen dieser Studie sind verschiedene Aspekte hervorzuheben. Obwohl eine validierte deutschsprachige Version des Grit Konstruktes eingesetzt wurde, zeigte die KFA, dass zumindest für die Erfassung auf der gymnasialen Oberstufe Optimierungspotenzial vorhanden ist. So wies die Dimension Beharrlichkeit ein Item mit einer geringen Trennschärfe auf (siehe für eine Diskussion auch Muenks et al. 2017). Im Gegensatz zu den SRL-Kompetenzen, haben wir Grit nicht domänenspezifisch erfasst. Leichte Anpassungen in den Formulierungen sowie eine kontextspezifischere Erfassung könnten hier zielführend sein (siehe Schmidt et al. 2017). Die Validität der Ergebnisse wird zudem dadurch eingeschränkt, dass die motivationalen Regulationsstrategien sowie die kognitiven Regulationskompetenzen einzig aus Sicht der Schülerinnen und Schüler und zudem nicht mit handlungsnahen 
Erhebungsverfahren erfasst wurden. Des Weiteren ist festzuhalten, dass die motivationalen Regulationsstrategien retrospektiv erfasst wurden und diesbezüglich die Gefahr besteht, dass sich die Schülerinnen und Schüler überschätzen und die retrospektive Erfassung eine geringe Übereinstimmung mit dem tatsächlichen Handeln hat. Darüber hinaus wäre es wünschenswert gewesen, als Leistungskriterium nicht einzig die durch die Lehrpersonen offiziell festgelegten Noten zu berücksichtigen, sondern eine objektivere kriteriale sowie schulübergreifende Beurteilung der Maturaarbeit einzubeziehen. In dem Sinne wäre es sinnvoll, die entsprechenden Analysen nicht nur schulübergreifend, sondern auch innerhalb der Schulen durchzuführen, was im vorliegenden Projekt durch eine zu geringe schulspezifische Stichprobengröße allerdings nicht möglich war. Aus einer inhaltlichen Perspektive ist weiter anzumerken, dass die berücksichtigen Variablen mit einer aufgeklärten Varianz von knapp $10 \%$ nur einen geringen Teil der Leistungsunterschiede zwischen den Schülerinnen und Schülern erklären konnten. In der Metaanalyse von Credé et al. (2017) trug ebenfalls Beharrlichkeit, nicht aber beständiges Interesse, mit lediglich 2 bis $8 \%$ nur gering zur inkrementellen Varianzaufklärung der Leistung bei. Es wäre deshalb notwendig, den Zusammenhang zwischen Grit und Leistung anhand komplexerer Modelle und weiteren Variablen zu untersuchen. Möglicherweise wäre die Berücksichtigung motivationaler Merkmale (z. B. Zielorientierungen, Selbstwirksamkeit) und Persönlichkeitseigenschaften (z.B. Gewissenhaftigkeit) ertragreich, da diese Dimensionen einen Einfluss auf die Leistung von Schülerinnen und Schülern ausüben (Abuhassan und Bates 2015; Credé et al. 2017). In der Literatur lassen sich unterschiedliche Zusammenhänge zwischen Grit und Leistung in Bezug auf die Schulstufe beobachten (Muenks et al. 2017; Steinmayr et al. 2018). Aus diesem Grund ist es wichtig, dass die hier berichteten Befunde für andere Schulstufen überprüft werden.

Trotz dieser Einschränkungen konnten unsere Ergebnisse aufzeigen, dass Grit - insbesondere Beharrlichkeit - eine wichtige Rolle zur Erklärung interindividuellen Unterschieden bei den SRL-Kompetenzen und der Leistung im Kontext einer längerfristigen und komplexen Aufgabe einnimmt. Grit kann folglich helfen, unterschiedliche individuelle Vorgehensweisen von Schülerinnen und Schüler beim Lösen von komplexen und längerfristigen Aufgaben zu erklären. Die Ergebnisse dieser Studie machen deutlich, dass für die Erreichung von schwierigen Zielen, auch bei vergleichbaren leistungsbezogenen Voraussetzungen, nicht nur kognitive Kompetenzen, sondern auch die Fähigkeit, diese Kompetenzen über die Zeit hinweg beharrlich einzusetzen, relevant ist. Weitere Studien sind jedoch notwendig, um Grit und seine Zusammenhänge mit anderen Merkmalen bei anspruchsvollen und längerfristigen Aufgaben umfassend und unter Kontrolle weiterer Merkmale kritisch zu untersuchen.

Open Access Dieser Artikel wird unter der Creative Commons Namensnennung 4.0 International Lizenz (http://creativecommons.org/licenses/by/4.0/deed.de) veröffentlicht, welche die Nutzung, Vervielfältigung, Bearbeitung, Verbreitung und Wiedergabe in jeglichem Medium und Format erlaubt, sofern Sie den/die ursprünglichen Autor(en) und die Quelle ordnungsgemäß nennen, einen Link zur Creative Commons Lizenz beifügen und angeben, ob Änderungen vorgenommen wurden. 


\section{Literatur}

Abuhassan, A., \& Bates, T.C. (2015). Grit distinguishing effortful persistence from conscientiousness. Journal of Individual Differences, 36(4), 205-214. https://doi.org/10.1027/1614-0001/a000175.

Arslan, S., Akin, A., \& Çitemel, N. (2013). The predictive role of grit on metacognition in turkisch university students. Studia Psychologica, 55(4), 311-320.

Artelt, C., \& Neuenhaus, N. (2010). Metakognition und Leistung. In W. Bos, E. Klieme \& O. Köller (Hrsg.), Schulische Lerngelegenheiten und Kompetenzentwicklung. Festschrift für Jürgen Baumert (S. 127-246). Münster: Waxmann.

Bazelais, P., Lemay, D. J., \& Doleck, T. (2016). How does grit impact college students' academic achievement in science? European Journal of Science and Mathematics Education, 4(1), 33-43.

Bowman, N. A., Hill, P.L., Denson, N., \& Bronkema, R. (2015). Keep on truckin' or stay the course? Exploring grit dimensions as differential predictors of educational achievement, satisfaction, and intentions. Social Psychological and Personality Science, 6(6), 639-645. https://doi.org/10.1177/ 1948550615574300.

Credé, M., \& Phillips, L. A. (2011). A meta-analytic review of the motivated strategies for learning questionnaire. Learn Individ Differ, 21(4), 337-346. https://doi.org/10.1016/j.lindif.2011.03.002.

Credé, M., Tynan, M.C., \& Harms, P.D. (2017). Much ado about grit: a meta-analytic synthesis of the grit literature. Journal of Personality and Social Psychology, 113(3), 492-511. https://doi.org/10.1037/ pspp0000102.

De Feyter, T., Caers, R., Vigna, C., \& Berings, D. (2012). Unraveling the impact of the Big Five personality traits on academic performance: The moderating and mediating effects of self-efficacy and academic motivation. Learning and Individual Differences, 22(4), 439-448. https://doi.org/10.1016/ j.lindif.2012.03.013.

Duckworth, A. L., \& Quinn, P. D. (2009). Development and validation of the short grit scale (grit-s). Journal of Personality Assessment, 91(2), 166-174. https://doi.org/10.1080/00223890802634290.

Duckworth, A.L., Peterson, C., Matthews, M.D., \& Kelly, D. R. (2007). Grit: perseverance and passion for long-term goals. Journal of Personality and Social Psychology, 92(6), 1087-1101. https://doi.org/10. 1037/0022-3514.92.6.1087.

Duckworth, A.L., Quinn, P.D., \& Seligman, M.E.P. (2009). Positive predictors of teacher effectiveness. The Journal of Positive Psychology, 4(6), 540-547. https://doi.org/10.1080/17439760903157232.

Duckworth, A. L., Kirby, T. A., Tsukayama, E., Berstein, H., \& Ericsson, K. A. (2011). Deliberate practice spells success: why grittier competitors triumph at the national spelling bee. Social Psychological and Personality Science, 2(2), 174-181. https://doi.org/10.1177/1948550610385872.

Dumfart, B., \& Neubauer, A.C. (2016). Conscientiousness is the most powerful noncognitive predictor of school achievement in adolescents. Journal of Individual Differences, 37(1), 8-15. https://doi.org/10. 1027/1614-0001/a000182.

Engelschalk, T., Steuer, G., \& Dresel, M. (2015). Wie spezifisch regulieren Studierende ihre Motivation bei unterschiedlichen Anlässen? Zeitschrift für Entwicklungspsychologie und Pädagogische Psychologie, 47(1), 14-23. https://doi.org/10.1026/0049-8637/a000120.

Eskreis-Winkler, L., Shulman, E.P., Beal, S.A., \& Duckworth, A.L. (2014). The grit effect: predicting retention in the military, the workplace, school and marriage. Frontiers in Psychology, 5, 36. https:// doi.org/10.3389/fpsyg.2014.00036.

Eskreis-Winkler, L., Shulman, E.P., Young, V., Tsukayama, E., Brunwasser, S. M., \& Duckworth, A.L. (2016). Using wise interventions to motivate deliberate practice. Journal of Personality and Social Psychology, 111(5), 728-744. https://doi.org/10.1037/pspp0000074.

Eskreis-Winkler, L., Gross, J. J., \& Duckworth, A. (2018). Grit: sustained self-regulation in the service of Superordinate goals. In K. D. Vohs \& R. F. Baumeister (Hrsg.), Handbook of self-regulation (3. Aufl. S. 380-395). New York: Guilford.

Fite, R.E., Lindeman, M.I.H., Rogers, A.P., Voyles, E., \& Durik, A.M. (2017). Knowing oneself and long-term goal pursuit: relations among self-concept clarity, conscientiousness, and grit. Personality and Individual Differences, 108, 191-194. https://doi.org/10.1016/j.paid.2016.12.008.

Fleckenstein, J., Schmidt, F. T.C., \& Möller, J. (2014). Wer hat Biss? Beharrlichkeit und beständiges Interesse von Lehramtsstudierenden. Eine deutsche Adaptation der 12-Item Grit Scale. Psychologie in Erziehung und Unterricht. https://doi.org/10.2378/peu2014.art22d.

Graham, S., \& Harris, K. R. (2000). The role of self-regulation and transcription skills in writing and writing development. Educational Psychologist, 35(1), 3-12. https://doi.org/10.1207/s15326985ep3501_ 2 . 
Händel, M., Artelt, C., \& Weinert, S. (2013). Assessing metacognitive knowledge: development and evaluation of a test instrument. Journal for Educational Research Online, 5(2), 162-188.

Hodge, B., Wright, B., \& Bennett, P. (2017). The role of grit in determining engagement and academic outcomes for university students. Research in Higher Education. https://doi.org/10.1007/s11162-0179474-y.

Karlen, Y. (2015). Nutzungshäufigkeit von Lernstrategien und metakognitives Strategiewissen in der Oberstufe des Gymnasiums: Entwicklung und Zusammenhänge. Zeitschrift für Bildungsforschung, 5(2), 159-175. https://doi.org/10.1007/s35834-015-0123-2.

Karlen, Y. (2017). The development of a new instrument to assess metacognitive strategy knowledge about academic writing and its relation to self-regulated writing and writing performance. Journal of Writing Research, 9(1), 61-86. https://doi.org/10.17239/jowr-2017.09.01.03.

Karlen, Y., Suter, F., \& Compagnoni, M. (in Vorb.). Selbstregulationskompetenzen im Studium: Entwicklung und Validierung von Skalen zu Erfassung von selbstregulationsbezogener Kompetenzen.

Komarraju, M., Karau, S. J., \& Schmeck, R. R. (2009). Role of the Big Five personality traits in predicting college students' academic motivation and achievement. Learning and Individual Differences, 19(1), 47-52. https://doi.org/10.1016/j.lindif.2008.07.001.

Lenzner, A., \& Dickhäuser, O. (2011). Strategien der Motivationsregulation: Theoretische Überlegungen und empirische Befunde. In M. Dresel \& L. Lämmle (Hrsg.), Motivation, Selbstregulation, Leistungsexellenz (S. 11-38). Münster: LIT.

Leopold, C., \& Leutner, D. (2002). Der Einsatz von Lernstrategien in einer konkreten Lernsituation bei Schülern unterschiedlicher Jahrgangsstufen. Zeitschrift für Pädagogik, 45, 240-258.

Maag Merki, K., Ramseier, E., \& Karlen, Y. (2013). Reliability and validity analyses of a newly developed test to assess learning strategy knowledge. Journal of Cognitive Education and Psychology, 12(3), 391-408. https://doi.org/10.1891/1945-8959.12.3.391.

MacKinnon, D. P., Lockwood, C. M., \& Williams, J. (2004). Confidence limits for the indirect effect: Distribution of the product and resampling methods. Multivariate Behavioral Research, 39(1), 99-128.

Muenks, K., Wigfield, A., Yang, J.S., \& O’Neal, C.R. (2017). How true is grit? Assessing its relations to high school and college students' personality characteristics, self-regulation, engagement, and achievement. Journal of Educational Psychology, 109(5), 599-620. https://doi.org/10.1037/edu0000153.

Muthén, L. K., \& Muthén, B. O. (2017). Mplus user's guide (8. Aufl.). Los Angeles: Muthén \& Muthén.

Neuenhaus, N., Artelt, C., Lingel, K., \& Schneider, W. (2010). Fifth graders metacognitive knowledge: General or domain-specific? European Journal of Psychology of Education, 26(2), 163-178. https:// doi.org/10.1007/s10212-010-0040-7.

Neuenhaus, N., Artelt, C., \& Schneider, W. (2017). Lernstrategiewissen im Bereich Englisch. Entwicklung und erste Validierung eines Tests für Schülerinnen und Schüler der frühen Sekundarstufe. Diagnostica, 63(2), 135-147. https://doi.org/10.1026/0012-1924/a000171.

Nota, L., Soresi, S., \& Zimmerman, B. J. (2004). Self-regulation and academic achievement and resilience: a longitudinal study. International Journal of Educational Research, 41(3), 198-215. https://doi.org/ 10.1016/j.ijer.2005.07.001.

Paris, S. G., Lipson, M. Y., \& Wixson, K. K. (1983). Becoming a strategic reader. Contemporary Educational Psychology, 8(3), 293-316. https://doi.org/10.1016/0361-476x(83)90018-8.

Pintrich, P. R. (2000). The role of goal orientation in self-regulated learning. In M. Boekaerts, P. R. Pintrich \& M. Zeidner (Hrsg.), Handbook of self-regulated learning (S. 451-502). San Diego: Academic Press.

Rimfeld, K., Kovas, Y., Dale, P.S., \& Plomin, R. (2016). True grit and genetics: Predicting academic achievement from personality. Journal of Personality and Social Psychology, 111(5), 780-789. https://doi. org/10.1037/pspp0000089.

Schermelleh-Engel, K., Moosbrugger, H., \& Müller, H. (2003). Evaluating the fit of structural equation models: tests of significance and descriptive goodness-of-fit measures. Methods of Psychological Research Online, 8(2), 23-74.

Schmidt, F.T.C., Fleckenstein, J., Retelsdorf, J., Eskreis-Winkler, L., \& Möller, J. (2017). Measuring grit: A German validation and a domain-specific approach to grit. European Journal of Psychological Assessment. Advance online publication. https://doi.org/10.1027/1015-5759/a000407.

Schwinger, M., von der Laden, T., \& Spinath, B. (2007). Strategien zur Motivationsregulation und ihre Erfassung. Zeitschrift für Entwicklungspsychologie und Pädagogische Psychologie, 39(2), 57-69. https://doi.org/10.1026/0049-8637.39.2.57.

Smit, K., de Brabander, C. J., Boekaerts, M., \& Martens, R. L. (2017). The self-regulation of motivation: Motivational strategies as mediator between motivational beliefs and engagement for learning. 
International Journal of Educational Research, 82, 124-134. https://doi.org/10.1016/j.ijer.2017.01. 006.

Steinmayr, R., Weidinger, A.F., \& Wigfield, A. (2018). Does students' grit predict their school achievement above and beyond their personality, motivation, and engagement? Contemporary Educational Psychology, 53, 106-122. https://doi.org/10.1016/j.cedpsych.2018.02.004.

Teng, L.S., \& Zhang, L.J. (2018). Effects of motivational regulation strategies on writing performance: A mediation model of self-regulated learning of writing in English as a second/foreign language. Metacognition and Learning, 13(2), 213-240. https://doi.org/10.1007/s11409-017-9171-4

Tyumeneva, Y., Kardanova, E., \& Kuzmina, J. (2017). Grit. Two related but independent constructs instead of one. Evidence from Item Response Theory. European Journal of Psychological Assessment. https://doi.org/10.1027/1015-5759/a000424.

Weinstein, C.E., Acee, T. W., \& Jung, J. (2011). Self-regulation and learning strategies. New Directions for Teaching and Learning, 2011(126), 45-53. https://doi.org/10.1002/t1.443.

Weisskirch, R.S. (2016). Grit, self-esteem, learning strategies and attitudes and estimated and achieved course grades among college students. Current Psychology, 37(1), 21-27. https://doi.org/10.1007/ s12144-016-9485-4.

West, M.R., Kraft, M.A., Finn, A.S., Martin, R.E., Duckworth, A.L., Gabrieli, C.F.O., \& Gabrieli, J.D.E. (2016). Promise and paradox: measuring students' non-cognitive skills and the impact of schooling. Educational Evaluation and Policy Analysis, 38(1), 148-170. https://doi.org/10.3102/ 0162373715597298.

Wigfield, A., \& Eccles, J.S. (2000). Expectancy-value theory of achievement motivation. Contemporary Educational Psychology, 25(1), 68-81. https://doi.org/10.1006/ceps.1999.1015.

Wolters, C. A. (2003). Regulation of motivation: evaluating an underemphasized aspect of self-regulated learning. Educational Psychologist, 38(4), 189-205. https://doi.org/10.1207/S15326985EP3804_1.

Wolters, C. A., \& Hussain, M. (2015). Investigating grit and its relations with college students' self-regulated learning and academic achievement. Metacognition and Learning, 10(3), 293-311. https://doi. org/10.1007/s11409-014-9128-9. 